Pure and Applied Mathematics Quarterly

Volume 7, Number 4

(Special Issue:

In memory of Eckart Viehweg)

1495-1515, 2011

\title{
Irrational Centers
}

\author{
Sándor Kovács \\ In memoriam Eckart Viehweg
}

\begin{abstract}
Irrational centers are defined analogously to associated primes. The union of irrational centers is the locus of non-rational singularities, but irrational centers carry more information. There may be embedded irrational centers signifying more complicated singularities. Various results regarding irrational centers are proved, in particular some concerning depth estimates and the Cohen-Macaulayness of certain ideal sheaves. It is also proved that absolute irrational centers of a log canonical pair are also non-klt centers. This allows applying results proved for irrational centers for non-klt centers of log canonical pairs.
\end{abstract}

Keywords: rational, log canonical, Cohen-Macaulay, non-klt centers.

\section{INTRODUCTION}

Rational singularities form one of the most important classes of singularities. Their essence lies in the fact that their cohomological behavior is very similar to that of smooth points. For instance, vanishing theorems can be easily extended

Received: Oct. 19, 2010; Revised: Mar. 2, 2011.

Supported in part by NSF Grant DMS-0856185, and the Craig McKibben and Sarah Merner Endowed Professorship in Mathematics at the University of Washington. 
to varieties with rational singularities. Establishing that a certain class of singularities is rational opens the door to using very powerful tools on varieties with those singularities.

The main purpose of the present article is to get a handle on determining how far a non-rational singularity is from being rational, or in other words, introduce a measure of the failure of a singularity being rational.

Recently there has been an effort to extend the notion of rational singularities to pairs. There are at least two approaches; Schwede and Takagi [ST08] are dealing with pairs $(X, \Delta)$ where $\lfloor\Delta\rfloor=0$ while Kollár and Kovács [KK09] are studying pairs $(X, \Delta)$ where $\Delta$ is reduced. I will work with pairs and concentrate on the latter approach, but the results are interesting already in the classical case and should be easily adjustable to fit the setup of the former approach.

I will introduce and start developing the notion of irrational centers (or nonrational centers). These are special subvarieties of the singular locus that are one way or another "responsible" for he failure of the singularity to be rational. After having finished this article I was informed that Alexeev and Hacon has introduced a similar notion in [AH09]. The definition given here reduces to their definition in the case $\Delta=0$. Some of their results are similar to the ones in the present article, but their methods are different from those applied here.

The behaviour of irrational centers is very similar to that of non-klt centers. In fact, I will show that Kollár's recent results [Kol10] (cf. [Ale08], [Fuj09]) concerning depth of ideal sheaves of unions of non-klt centers has a reasonably close analogue for irrational centers. In particular, I will prove the following results (for the relevant definitions, see $\S 2$ and $\S 4$ ):

Theorem 1.1 (= Corollary 3.4). Let $(X, D)$ be a rational pair. Then $\mathscr{O}_{X}(-D)$ is a CM sheaf.

Theorem 1.2 (= Corollary 5.4). Let $(X, D)$ be a normal pair and $x \in X$ which is not the general point of an absolute irrational center of $(X, D)$. Then

$$
\operatorname{depth}_{x} \mathscr{O}_{X}(-D) \geq \min \left(3, \operatorname{codim}_{X} x\right)
$$

The main focus of this article is the introduction of the notion of irrational centers as a tool to study singularities. The above theorem is a demonstration of how one may use this notion. I also prove that 
Theorem 1.3 (= Theorem 6.3). Absolute irrational centers of lc pairs are also non-klt centers.

This opens the door to numerous possible applications regarding log canonical pairs. For instance it implies the following:

Theorem 1.4 (= Corollary 3.5) [KK09, Thm. 111],[Kol10, Thm. 2]. Let $(X, \Delta)$ be a dlt pair. Then for any effective integral divisor $D \leq\lfloor\Delta\rfloor \mathscr{O}_{X}(-D)$ is a $C M$ sheaf.

Theorem 1.5 (= Corollary 6.4) [Ale08],[Fuj09],[Kol10]. Let $(X, \Delta)$ be an lc pair and $x \in X$ which is not a non-klt center of $(X, \Delta)$. Then

$$
\operatorname{depth}_{x} \mathscr{O}_{X}(-\lfloor\Delta\rfloor) \geq \min \left(3, \operatorname{codim}_{X} x\right) .
$$

It should also be noted that for lc pairs Kollár's results are more general than the ones here. On one hand his results extend to more generally chosen integral divisors and also to slc singularities. I believe both of those generalizations are possible through the methods presented here, but this will be addressed at a later time.

Definitions and Notation 1.6. Unless otherwise stated, all objects are assumed to be defined over $\mathbb{C}$, all schemes are assumed to be of finite type over $\mathbb{C}$ and a morphism means a morphism between schemes of finite type over $\mathbb{C}$.

For definitions related to pairs, see (2.A).

If $\phi: Y \rightarrow Z$ is a birational morphism, then $\operatorname{Exc}(\phi)$ will denote the exceptional set of $\phi$. By abuse of notation, if this exceptional set is of pure codimension 1, then $\operatorname{Exc}(\phi)$ will also denote the exceptional divisor of $\phi$. For a closed subscheme $W \subseteq X$, the ideal sheaf of $W$ is denoted by $\mathscr{I}_{W \subseteq X}$ or if no confusion is likely, then simply by $\mathscr{I}_{W}$. For a point $x \in X, \kappa(x)$ denotes the residue field of $\mathscr{O}_{X, x}$.

For a proper birational morphism $\pi: Y \rightarrow X$ let $T \subseteq X$ denote the indeterminacy locus of the rational map $\pi^{-1}: X \rightarrow Y$. Then for a subset $W \subseteq X$ we define the strict transform of $W$ on $Y$, denoted by $\pi_{*}^{-1} W$ as the closure of $\pi^{-1}(W \backslash T)$ in $Y$. Notice that if $W$ is contained in $T$, then its strict transform is the empty set.

Let $X$ be a noetherian scheme, $x \in X$ a (not necessarily closed) point, and $\mathscr{F}$ a coherent sheaf on $X$. The dimension and codimension of the closed subscheme 
$\overline{\{x\}} \subseteq X$ will be denoted by $\operatorname{dim} x$ and $\operatorname{codim}_{X} x$ respectively. In particular, $\operatorname{dim} X=\operatorname{dim} x+\operatorname{codim}_{X} x$ for any $x \in X$. The dimension of $\mathscr{F}$ is the dimension of its support: $\operatorname{dim} \mathscr{F}:=\operatorname{dim} \operatorname{supp} \mathscr{F}$. The local dimension, denoted by $\operatorname{dim}_{x}$ is understood on the local scheme $(X, x)$ and it is equal to $\operatorname{dim} \mathscr{F}_{x}$ the dimension of $\mathscr{F}_{x}$ as an $\mathscr{O}_{X, x}$-module. The depth of $\mathscr{F}$ at $x$, denoted by depth $\mathscr{F}$ is defined as the depth of $\mathscr{F}_{x}$ as an $\mathscr{O}_{X, x}$-module. A non-zero coherent sheaf $\mathscr{F}$ is said to satisfy Serre's condition $S_{n}$ if

$$
\operatorname{depth}_{x} \mathscr{F} \geq \min \left(n, \operatorname{dim}_{x} \mathscr{F}\right)
$$

for all $x \in X$ [BH93, p.63]. Notice that this definition implies that if $X$ is contained in another noetherian scheme $Y$, then $\mathscr{F}$ satisfies Serre's condition $S_{n}$ regarded as a sheaf on $X$ if and only if it satisfies $S_{n}$ regarded as a sheaf on $Y$. This is because the depth as well as the support of $\mathscr{F}$ is independent of the ambient scheme considered.

The dualizing complex of $X$ is denoted by $\omega_{X}^{\circ}$ and if $X$ is of pure dimension $n$ the dualizing sheaf of $X$ is defined as $\omega_{X}:=h^{-n}\left(\omega_{X}^{*}\right)$. Note that if $X$ is not normal, then this is not necessarily the push-forward of the canonical sheaf from the non-singular locus.

Let $x \in X$ be a closed point. Then $\mathscr{F}$ is called Cohen-Macaulay (CM for short) at $x$ if $\mathscr{F}_{x}$ is a Cohen-Macaulay module over $\mathscr{O}_{X, x}$, and $\mathscr{F}$ is Cohen-Macaulay ( $C M$ for short) if it is $\mathrm{CM}$ at $x$ for all closed points $x \in \operatorname{supp} \mathscr{F}$. In particular, $X$ is CM if so is $\mathscr{O}_{X}$. Finally, $X$ is called Gorenstein if $\mathscr{O}_{X}$ is CM and $\omega_{X}$ is an invertible sheaf.

A relatively straightforward consequence of the definition of the dualizing sheaf and basic properties of CM rings is that $X$ is CM if and only if $\omega_{X}{ } \simeq_{\text {qis }} \omega_{X}[n]$ cf. [Con00, 3.5.1].

Let $X$ be a complex scheme of dimension n. Let $D_{\text {filt }}(X)$ denote the derived category of filtered complexes of $\mathscr{O}_{X}$-modules with differentials of order $\leq 1$ and $D_{\text {filt,coh }}(X)$ the subcategory of $D_{\text {filt }}(X)$ of complexes $\mathrm{K}$, such that for all $i$, the cohomology sheaves of $G r_{\text {filt }}^{i} \mathrm{~K}$ are coherent cf. [DB81], [GNPP88]. Let $D(X)$ and $D_{\text {coh }}(X)$ denote the derived categories with the same definition except that the complexes are assumed to have the trivial filtration. The superscripts,,$+- b$ carry the usual meaning (bounded below, bounded above, bounded). Isomorphism in these categories is denoted by $\simeq_{\text {qis }}$. A sheaf $\mathscr{F}$ is also considered as a 
complex $\mathscr{F}^{\bullet}$ with $\mathscr{F}^{0}=\mathscr{F}$ and $\mathscr{F}^{i}=0$ for $i \neq 0$. If $\mathrm{K}$ is a complex in any of the above categories, then $h^{i}(\mathrm{~K})$ denotes the $i$-th cohomology sheaf of $\mathrm{K}$.

The right derived functor of an additive functor $F$, if it exists, is denoted by $R F$ and $\mathcal{R}^{i} F$ is short for $h^{i} \circ \mathcal{R} F$. Furthermore, $\mathbb{H}^{i}, \mathbb{H}_{\mathrm{c}}^{i}, \mathbb{H}_{Z}^{i}$, and $\mathscr{H}_{Z}^{i}$ will denote $\mathcal{R}^{i} \Gamma$, $\mathcal{R}^{i} \Gamma_{\mathrm{c}}, \mathcal{R}^{i} \Gamma_{Z}$, and $\mathcal{R}^{i} \mathscr{H}_{Z}$ respectively, where $\Gamma$ is the functor of global sections, $\Gamma_{\mathrm{c}}$ is the functor of global sections with proper support, $\Gamma_{Z}$ is the functor of global sections with support in the closed subset $Z$, and $\mathscr{H}_{Z}$ is the functor of the sheaf of local sections with support in the closed subset $Z$. Note that according to this terminology, if $\phi: Y \rightarrow X$ is a morphism and $\mathscr{F}$ is a coherent sheaf on $Y$, then $\mathcal{R} \phi_{*} \mathscr{F}$ is the complex whose cohomology sheaves give rise to the usual higher direct images of $\mathscr{F} \cdot \mathcal{H} m_{X}(\mathscr{F}, \mathscr{G})$ denotes the sheaf of morphisms between the sheaves $\mathscr{F}$ and $\mathscr{G}$ and $\mathcal{E} x t_{X}^{i}:=\mathcal{R}^{i} \mathcal{H o m}_{X}=h^{i} \circ \mathcal{R} \mathcal{H o m}_{X}$.

We will use the notion that a morphism $f: \mathrm{A} \rightarrow \mathrm{B}$ in a derived category has a left inverse. This means that there exists a morphism $f^{\ell}: B \rightarrow A$ in the same derived category such that $f^{\ell} \circ f: \mathrm{A} \rightarrow \mathrm{A}$ is the identity morphism of A. I.e., $f^{\ell}$ is a left inverse of $f$.

\section{RATIONAL PAIRS}

\section{A. Basic definitions}

A $\mathbb{Q}$-divisor is a $\mathbb{Q}$-linear combination of integral Weil divisors; $\Delta=\sum a_{i} \Delta_{i}$, $a_{i} \in \mathbb{Q}, \Delta_{i}$ (integral) Weil divisor. For a $\mathbb{Q}$-divisor $\Delta=\sum a_{i} \Delta_{i}$ we will use the following notation: $\Delta_{\text {red }}:=\sum \Delta_{i}$ and the round-down of $\Delta$ is defined by the formula: $\lfloor\Delta\rfloor=\sum\left\lfloor a_{i}\right\rfloor \Delta_{i}$, where $\left\lfloor a_{i}\right\rfloor$ is the largest integer not larger than $a_{i}$.

A log variety or pair $(X, \Delta)$ consists of an irreducible variety (i.e., an irreducible reduced scheme of finite type over a field $k) X$ and an effective $\mathbb{Q}$ divisor $\Delta \subseteq X$ such that no irreducible component of $\Delta$ is contained in $\operatorname{Sing} X$. (This last assumption is automatically satisfied if, for example, $X$ is normal.) A morphism of pairs $\phi:(Y, \Gamma) \rightarrow(X, \Delta)$ is a morphism $\phi: Y \rightarrow X$ such that $\phi(\operatorname{supp} \Gamma) \subseteq \operatorname{supp} \Delta$.

A reduced pair is a pair $(X, D)$ where $D$ is a reduced integral divisor. In this case with a slight abuse of notation we will use $D$ to also denote $\operatorname{supp} D$. If $(X, D)$ is a reduced pair, then $(X, D)$ is said to have simple normal crossings or to be an snc pair at $p \in X$ if $X$ is smooth at $p$ and the components of $D$ are 
smooth at $p$ intersecting transversely in a Zariski neighbourhood of $p$, i.e., in local analytic coordinates, $x_{1}, \ldots, x_{n}$, near $p, D$ is defined by $\prod_{i=1}^{r} x_{i}=0$ for some $r$. Furthermore, $(X, D)$ is snc if it is snc at every $p \in X$. An snc pair $(X, D)$ will be called a smooth pair if the irreducible components of $D$ do not intersect. Equivalently, $(X, D)$ is a smooth pair if and only if both $X$ and $D$ are smooth.

A morphism of pairs $\phi:\left(Y, \Delta_{Y}\right) \rightarrow(X, \Delta)$ is a partial log resolution of $(X, \Delta)$ if $\phi: Y \rightarrow X$ is a proper, birational morphism which is an isomorphism near general points of $\Delta$ such that $\Delta_{Y}=\phi_{*}^{-1} \Delta$. A partial log resolution is a $\log$ resolution if $\operatorname{Exc}(\phi)$ is a divisor and $\left(Y,\left(\Delta_{Y}\right)_{\text {red }}+\operatorname{Exc}(\phi)\right)$ is an snc pair. Note that we allow $(X, \Delta)$ to be snc and still call a morphism with these properties a log resolution. Also note that the notion of a log resolution is not used consistently in the literature.

If $(X, \Delta)$ is a pair, then $\Delta$ is called a boundary if $\lfloor(1-\varepsilon) \Delta\rfloor=0$ for all $0<\varepsilon<1$, i.e., the coefficients of all irreducible components of $\Delta$ are in the interval $[0,1]$. For the definition of $k l t$, $d l t$, and $l c$ pairs see [KM98]. Let $(X, \Delta)$ be a pair and $\mu: X^{\mathrm{m}} \rightarrow X$ a proper birational morphism. Let $E=\sum a_{i} E_{i}$ be the discrepancy divisor, i.e., a linear combination of exceptional divisors such that

$$
K_{X^{\mathrm{m}}}+\mu_{*}^{-1} \Delta \sim_{\mathbb{Q}} \mu^{*}\left(K_{X}+\Delta\right)+E
$$

and let $\Delta^{\mathrm{m}}:=\mu_{*}^{-1} \Delta+\sum_{a_{i} \leq-1} E_{i}$. For an irreducible divisor $F$ on a birational model of $X$ we define its discrepancy as its coefficient in $E$. Notice that as divisors correspond to valuations, this discrepancy is independent of the model chosen, it only depends on the divisor. A non-klt place of a pair $(X, \Delta)$ is an irreducible divisor $F$ over $X$ with discrepancy at most -1 and a non-klt center is the image of a non-klt place. $\operatorname{Exc}_{n k l t}(\mu)$ denotes the union of the loci of all non-klt places of $\phi$.

Note that in the literature, non-klt places and centers are often called log canonical places and centers. For a more detailed and precise definition see [HK10, p.37].

Now if $\left(X^{\mathrm{m}}, \Delta^{\mathrm{m}}\right)$ is as above, then it is a minimal dlt model of $(X, \Delta)$ if it is a dlt pair and the discrepancy of every $\mu$-exceptional divisor is at most -1 cf. [KK10]. Note that if $(X, \Delta)$ is lc with a minimal dlt model $\left(X^{\mathrm{m}}, \Delta^{\mathrm{m}}\right)$, then $K_{X^{\mathrm{m}}}+\Delta^{\mathrm{m}} \sim_{\mathbb{Q}} \mu^{*}\left(K_{X}+\Delta\right)$. Also note that minimal dlt models are not unique; 
for instance, blowing up the intersection of two or more irreducible components of an snc divisor produces a (new) minimal dlt model of the given snc pair.

\section{B. Rational pairs}

Recall the definitions of rational singularities:

Definition 2.1. Let $X$ be a normal variety and $\phi: Y \rightarrow X$ a resolution of singularities. $X$ is said to have rational singularities if $\mathcal{R}^{i} \phi_{*} \mathscr{O}_{Y}=0$ for all $i>0$, or equivalently if the natural map $\mathscr{O}_{X} \rightarrow \mathcal{R} \phi_{*} \mathscr{O}_{Y}$ is a quasi-isomorphism.

Definition 2.2. Let $(X, D)$ be a pair and $D$ an integral divisor. Then $(X, D)$ is called a normal pair if there exists a log resolution $\phi:\left(Y, D_{Y}\right) \rightarrow(X, D)$ such that the natural morphism $\phi^{\#}: \mathscr{O}_{X}(-D) \rightarrow \phi_{*} \mathscr{O}_{Y}\left(-D_{Y}\right)$ is an isomorphism.

Proposition 2.3. Let $(X, D)$ be a normal pair. Then $\left(X, D_{\text {red }}\right)$ is an snc pair in codimension 1 .

Proof. Let $\phi:\left(Y, D_{Y}\right) \rightarrow(X, D)$ be a $\log$ resolution for which the natural morphism

$$
\phi^{\#}: \mathscr{O}_{X}(-D) \rightarrow \phi_{*} \mathscr{O}_{Y}\left(-D_{Y}\right)
$$

is an isomorphism.

First consider $X \backslash \operatorname{supp} D$. Then $\phi^{\#}: \mathscr{O}_{X \backslash \operatorname{supp} D} \rightarrow \phi_{*} \mathscr{O}_{Y \backslash \operatorname{supp} \phi^{-1}(D)}$ is an isomorphism, and hence $X \backslash \operatorname{supp} D$ is normal. In particular, $X$ is smooth in codimension 1 away from $D$.

Next observe that by definition $\phi$ is an isomorphism near the general points of $D$ and hence $\left(X, D_{\text {red }}\right)$ is an snc pair in codimension 1 near $D$. This proves the claim.

Proposition 2.4. Let $(X, D)$ be a reduced pair. Then we have the following two implications:

(2.4.1) if $X$ is normal and $\mathcal{R}^{1} \phi_{*} \mathscr{O}_{Y}\left(-D_{Y}\right)=0$ for a log resolution $\phi:\left(Y, D_{Y}\right) \rightarrow(X, D)$, then $(X, D)$ is a normal pair, and

(2.4.2) if $(X, D)$ is a normal pair and $D$ is Cartier, then $X$ is normal. 
Proof. Consider the following diagram of exact sequences:

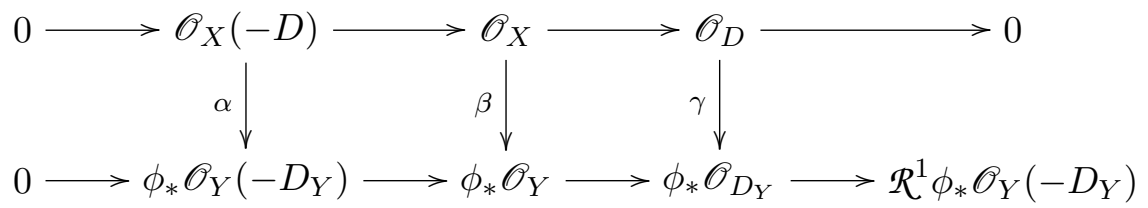

By definition both $X$ and $D$ are reduced, so $\beta$ and $\gamma$ are injective and then $\alpha$ is injective as well.

Recall that $X$ is normal if and only is $\beta$ is an isomorphism and $(X, D)$ is normal if and only is $\alpha$ is an isomorphism.

If $\beta$ is an isomorphism, then it is in particular surjective and hence $\gamma$ is surjective. If furthermore $\mathcal{R}^{1} \phi_{*} \mathscr{O}_{Y}\left(-D_{Y}\right)=0$, then $\gamma$ is an isomorphism and then by the Snake Lemma $\alpha$ is also an isomorphism. This proves (2.4.1).

If $\alpha$ is an isomorphism and $D$ is Cartier then $\phi^{*} D \sim D_{Y}+F$ for some effective $\phi$-exceptional divisor $F \subset Y$. Therefore $\alpha$ factors as

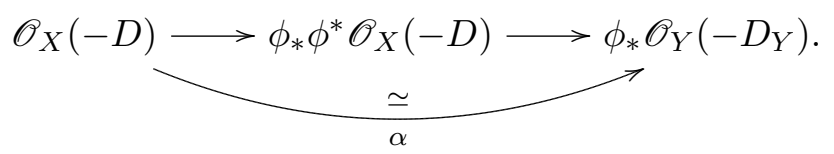

Since these are torsion-free sheaves of rank 1 it follows that all three must be isomorphic. In other words,

$$
\mathscr{O}_{X}(-D) \simeq \phi_{*} \phi^{*} \mathscr{O}_{X}(-D) \simeq \mathscr{O}_{X}(-D) \otimes \phi_{*} \mathscr{O}_{Y}
$$

and hence $\beta$ is an isomorphism, so (2.4.2) is proven.

Definition 2.5. [KK09] A reduced pair $(X, D)$ is called a rational pair if there exists a $\log$ resolution $\phi:\left(Y, D_{Y}\right) \rightarrow(X, D)$ such that

$$
\mathscr{O}_{X}(-D) \simeq_{\text {qis }} \mathcal{R} \phi_{*} \mathscr{O}_{Y}\left(-D_{Y}\right) .
$$

A log resolution as above will be called a rational log resolution of $(X, D)$.

REMARK 2.6. From the definition it is not obvious whether $(X, D)$ being rational implies that $X$ has rational singularities. It turns out that this is actually true if either $X$ is Cohen-Macaulay or $D$ is Cartier (2.12).

REMARK 2.7. Notice that this definition is different from that of Schwede and Takagi's [ST08] in several ways. In particular, according to this definition snc pairs are rational. On the other hand this definition is not independent of the 
resolution chosen but the only way it depends on the resolution is exactly the fact that it allows snc pairs to be rational. This situation is similar to the case of dlt singularities, where a dlt pair may have log canonical centers even though it has no "purely" log canonical singularities. In fact, the irrational centers (cf. (4.2)) are exactly the log canonical (or non-klt) centers of a dlt pair.

In Schwede and Takagi's terminology a rational pair with $D$ Cartier is said to have purely rational singularities. See [ST08, 3.15] for details.

ExAmple 2.8. Let $(X, \Delta)$ be a $\mathbb{Q}$-factorial dlt pair. Then it follows from [KK09, $111]$ or $[\operatorname{Kov} 10,2.1]$ that $(X,\lfloor\Delta\rfloor)$ is a rational pair.

The following is a simple consequence of known vanishing theorems that appear in various forms in [Amb03, Fuj09, KK09]. Since only this simple version is needed here, a reasonably self-contained proof is provided for the convenience of the reader. This statement was independently observed by Zsolt Patakfalvi.

Theorem 2.9 (Grauert-Riemenschneider vanishing for pairs) cf. [Amb03, Fuj09, KK09]. Let $(X, D)$ be a pair and $\phi:\left(Y, D_{Y}\right) \rightarrow(X, D)$ a log resolution. Let $B \leq D_{Y}$ be an effective reduced integral divisor. Then

$$
\mathcal{R}^{i} \phi_{*} \omega_{Y}(B)=0 \quad \text { for } i>0 .
$$

REMARK 2.9.1. Notice that the statement does not say that vanishing holds for any pairs. It is important that $D_{Y}$ is the strict transform of $D$.

We need the following simple generalization of the (relative) KawamataViehweg vanishing theorem. It is contained implicitly in [Amb03] and explicitly in [Fuj09, 2.33] and [KK09, 4.3].

Lemma 2.10. Let $Y$ be a smooth variety, $\phi: Y \rightarrow X$ a proper morphism, and $M a \phi$-nef and $\phi$-big $\mathbb{Q}$-divisor on $Y$. Further let $L$ be a Cartier divisor with $\mathscr{L}=\mathscr{O}_{Y}(L)$ and $B+\sum \Delta_{i}$ an effective simple normal crossing divisor on $Y$. Assume that for some $0 \leq a_{i}<1, L \equiv M+\sum a_{i} \Delta_{i}$ and that $M$ is $\phi$-big on all log canonical centers of $(Y, B)$. Then

$$
\mathcal{R}^{i} f_{*}\left(\omega_{Y}(B) \otimes \mathscr{L}\right)=0 \quad \text { for } i>0 .
$$

Proof. Write $B=B_{1}+B^{\prime}$ where $B_{1}$ is irreducible and consider the following short exact sequence,

$$
\left.0 \rightarrow \omega_{Y}\left(B^{\prime}\right) \otimes \mathscr{L} \rightarrow \omega_{Y}(B) \otimes \mathscr{L} \rightarrow \omega_{B_{1}}\left(\left.B^{\prime}\right|_{B_{1}}\right) \otimes \mathscr{L}\right|_{B_{1}} \rightarrow 0 .
$$


Notice that the $\log$ canonical centers of $\left(B_{1},\left.B^{\prime}\right|_{B_{1}}\right)$ are restrictions of the log canonical centers of $(Y, B)$. Therefore, using the long exact cohomology sequence of $\mathcal{R} \phi_{*}$ and induction on the number of components of $B$ and the dimension of $Y$ this reduces the statement to the usual Kawamata-Viehweg vanishing theorem.

Proof of (2.9). Since $\phi:\left(Y, D_{Y}\right) \rightarrow(X, D)$ is a $\log$ resolution, it follows that $D_{Y}+E$ is supported on an snc divisor where $E=\operatorname{Exc}(\phi)$ is the exceptional divisor of $\phi$. Therefore any $\log$ canonical center of $(Y, B)$, in other words any intersection of the irreducible components of $B$, intersects $E$ transversally. In particular $\mathscr{O}_{Y}$ is $\phi$-big on any log canonical center of $(Y, B)$ and hence the statement follows from (2.10).

Corollary 2.11. Let $(X, D)$ be a rational pair and $\phi:\left(Y, D_{Y}\right) \rightarrow(X, D)$ a rational resolution.

(2.11.1) $\mathscr{O}_{X}(-D) \simeq \phi_{*} \mathscr{O}_{Y}\left(-D_{Y}\right)$, i.e., $(X, D)$ is normal,

(2.11.2) $\mathcal{R}^{i} \phi_{*} \mathscr{O}_{Y}\left(-D_{Y}\right)=0$ for $i>0$,

(2.11.3) $\mathcal{R}^{i} \phi_{*} \omega_{Y}\left(D_{Y}\right)=0$ for $i>0$.

Theorem 2.12. Let $(X, D)$ be a rational pair and $\phi:\left(Y, D_{Y}\right) \rightarrow(X, D)$ a rational resolution. Then

$$
\begin{aligned}
& \phi_{*} \omega_{Y}\left(D_{Y}\right) \simeq \omega_{X}(D):=\mathcal{H o m}_{X}\left(\mathscr{O}_{X}(-D), \omega_{X}\right), \text { and } \\
& \phi_{*} \omega_{Y} \simeq \omega_{X}
\end{aligned}
$$

Proof. Let $n=\operatorname{dim} X$. Then Grothendieck duality and (2.11.3) yields that

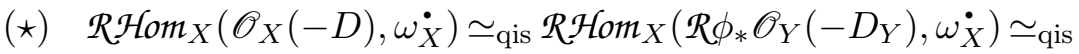

$$
\simeq_{\text {qis }} \mathcal{R} \phi_{*} \mathcal{R} \mathcal{H o m}_{Y}\left(\mathscr{O}_{Y}\left(-D_{Y}\right), \omega_{Y}^{\bullet}\right) \simeq_{\mathrm{qis}} \mathcal{R} \phi_{*} \omega_{Y}\left(D_{Y}\right)[n] \simeq_{\mathrm{qis}} \phi_{*} \omega_{Y}\left(D_{Y}\right)[n]
$$

and

$$
\omega_{D} \simeq_{\text {qis }} \mathcal{R} \mathcal{H o m}_{X}\left(\mathscr{O}_{D}, \omega_{X}\right) .
$$

Observe that $(\star)$ immediately implies (2.12.1). Consider the short exact sequence

$$
0 \rightarrow \mathscr{O}_{X}(-D) \rightarrow \mathscr{O}_{X} \rightarrow \mathscr{O}_{D} \rightarrow 0
$$

and apply the functor $\mathcal{R} \mathcal{H} m_{X}\left(-, \omega_{X}^{*}\right)$ to obtain the distinguished triangle

$$
\mathcal{R H o m}_{X}\left(\mathscr{O}_{D}, \omega_{X}^{\cdot}\right) \longrightarrow \omega_{X}^{\cdot} \longrightarrow \mathcal{R} \mathcal{H o m}_{X}\left(\mathscr{O}_{X}(-D), \omega_{X}^{\cdot}\right) \stackrel{+1}{\longrightarrow} \cdot
$$


By $(\star)$ and $(\star \star)$ this is the same as

$$
\omega_{D}^{\cdot} \longrightarrow \omega_{X}^{\cdot} \longrightarrow \phi_{*} \omega_{Y}\left(D_{Y}\right)[n] \stackrel{+1}{\longrightarrow} .
$$

Next consider the long exact sequence of cohomology sheaves induced by this distinguished triangle:

$$
0 \rightarrow \omega_{X} \rightarrow \phi_{*} \omega_{Y}\left(D_{Y}\right) \rightarrow \omega_{D} \rightarrow \ldots
$$

Since $Y$ is smooth we have a similar short exact sequence on $Y$.

$$
0 \rightarrow \omega_{Y} \rightarrow \omega_{Y}\left(D_{Y}\right) \rightarrow \omega_{D_{Y}} \rightarrow 0
$$

Applying $\phi_{*}$ and Grauert-Riemenschneider vanishing one obtains a commutative diagram of exact sequences:

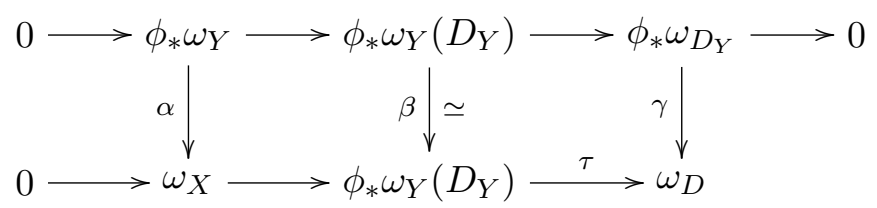

Since $\phi$ is an isomorphism at the general points of $Y$ and the irreducible components of $D_{Y}$ and since $\omega_{Y}$ and $\omega_{D_{Y}}$ are torsion-free, it follows that both $\alpha$ and $\gamma$ are injective. Then the fact that $\beta$ is an isomorphism implies that the image of $\tau$ is $\phi_{*} \omega_{D_{Y}} \subseteq \omega_{D}$. However, that implies that then $\operatorname{ker} \tau \simeq \phi_{*} \omega_{Y}$ and hence $\alpha$ is an isomorphism.

Corollary 2.13 [ST08, 3.20]. Let $(X, D)$ be a rational pair. Assume that $D$ is a Cartier divisor. Then $X$ has only rational singularities and in particular it is Cohen-Macaulay.

Proof. If $D$ is Cartier, then $h^{i}\left(\omega_{X}^{\cdot}\right)=0$ for $i \neq \operatorname{dim} X$ by $(\star)$ and hence $X$ is Cohen-Macaulay. It also follows that $X$ is normal by (2.4.2) and hence satisfies Kempf's criterion for rational singularities by (2.12.2).

This proves the statement, but actually one can give a simple direct proof:

Let $\phi:\left(Y, D_{Y}\right) \rightarrow(X, D)$ be a rational resolution and $n=\operatorname{dim} X$. By assumption the natural map $\mathscr{O}_{X}(-D) \rightarrow \mathcal{R} \phi_{*} \mathscr{O}_{Y}\left(-D_{Y}\right)$ is a quasi-isomorphism and since $\phi^{*} D-D_{Y}$ is an effective (exceptional) divisor it factors the following way:

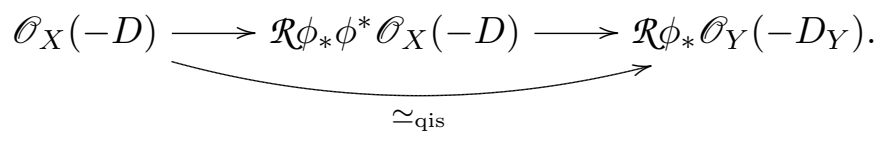


Twisting by $\mathscr{O}_{X}(D)$ implies that the natural morphism $\mathscr{O}_{X} \rightarrow \mathfrak{R} \phi_{*} \mathscr{O}_{Y}$ has a left inverse. Then $X$ has rational singularities by [Kov00, Thm. 1].

\section{DEPTH AND DUALITY}

The main statement in this section is a simple reformulation of Grothendieck's vanishing and non-vanishing theorems of certain local cohomology groups characterizing depth in terms of similar vanishing and non-vanishing involving the dualizing complex.

Throught the article dualizing complexes will be considered normalized so if $X$ is generically non-reduced then $\operatorname{supp} h^{i}\left(\omega_{X}^{\bullet} \subseteq \subseteq \operatorname{Sing} X\right.$ for $i \neq-\operatorname{dim} X$.

First we need an auxiliary result on the localization of dualizing complexes. This is undoubtedly known to experts. A proof is included for the benefit of the uninitiated reader.

Lemma 3.1. Let $X$ be a scheme that admits a dualizing complex $\omega_{X}^{\cdot}$ (this holds for instance if $X$ is of finite type over a field). Let $X_{x} \simeq$ Spec $\mathscr{O}_{X, x}$ denote the local scheme of $X$ at $x$. Then $X_{x}$ admits a dualizing complex and

$$
\omega_{X_{x}} \simeq \omega_{X}^{\cdot} \otimes \mathscr{O}_{X, x}[-\operatorname{dim} x]
$$

Proof. As the statement is local we may assume that $X$ is embedded into a Gorenstein scheme as a closed subscheme by [Kaw02, 1.4]. Let $j: X \hookrightarrow Y$ be such an embedding, $N=\operatorname{dim} Y$ and $m=\operatorname{codim}_{Y} x=\operatorname{dim} \mathscr{O}_{Y, x}$. Then by Grothendieck duality and because $Y$ is CM and $j$ is a closed embedding,

$$
\begin{aligned}
& \omega_{X}^{\cdot} \simeq_{\text {qis }} \mathcal{R} \mathcal{H o m}_{X}\left(\mathscr{O}_{X}, \omega_{X}^{\cdot}\right) \simeq_{\text {qis }} \mathcal{R} j_{*} \mathcal{R H o m} \mathcal{H}_{X}\left(\mathscr{O}_{X}, \omega_{X}^{\cdot}\right) \simeq_{\text {qis }} \\
& \underbrace{\simeq_{\text {qis }}}_{\text {Grothendieck duality }} \mathcal{R H o m} \mathcal{H}_{Y}(\mathcal{R} j_{*} \mathscr{O}_{X}, \underbrace{\omega_{\dot{Y}}}_{\text {dualizing complex }}) \simeq_{\text {qis }} \mathcal{R H o m} \mathcal{H}_{Y}(\mathscr{O}_{X}, \underbrace{\omega_{Y}}_{\text {dualizing sheaf }})[N] .
\end{aligned}
$$

Then taking cohomology and localizing at $x$ gives that

$$
\begin{aligned}
\omega_{X}^{\cdot} & \otimes \mathscr{O}_{X, x}[-\operatorname{dim} x] \simeq_{\mathrm{qis}}\left(\omega_{X}^{\cdot}\right)_{x}[-\operatorname{dim} x] \simeq_{\mathrm{qis}} \\
& \simeq_{\mathrm{qis}}\left(\mathcal{R} \mathcal{H o m}_{Y}\left(\mathscr{O}_{X}, \omega_{Y}\right)[N-\operatorname{dim} x]\right)_{x} \simeq_{\mathrm{qis}} \mathcal{R} \mathcal{H o m}_{Y_{x}}\left(\mathscr{O}_{X, x}, \omega_{Y, x}[m]\right) \simeq_{\mathrm{qis}} \\
& \simeq_{\mathrm{qis}} \mathcal{R} \mathcal{H o m}_{Y_{x}}\left(\mathcal{R}\left(j_{x}\right)_{*} \mathscr{O}_{X, x}, \omega_{Y, x}\right) \simeq_{\mathrm{qis}} \mathcal{R}\left(j_{x}\right)_{*} \mathcal{R} \mathcal{H}_{\mathrm{H}_{X}}\left(\mathscr{O}_{X, x}, \omega_{X, x}\right) \simeq_{\mathrm{qis}} \omega_{X, x}
\end{aligned}
$$


Proposition 3.2. Let $X$ be a scheme that admits a dualizing complex $\omega_{X}^{\cdot}$. Let $x \in X$ be a point and $\mathscr{F}$ a coherent sheaf on $X$. Let $d=\operatorname{dim}_{x} \mathscr{F}+\operatorname{dim} x$ and $t=\operatorname{depth}_{x} \mathscr{F}+\operatorname{dim} x$. Then

$$
\left(\mathcal{E} x t_{X}^{-i}\left(\mathscr{F}, \omega_{X}\right)\right)_{x}=0 \quad \text { for } i>d \text { and } i<t .
$$

Furthermore, if $\mathscr{F}_{x} \neq 0$, then

$$
\left(\mathcal{E} x t_{X}^{-d}\left(\mathscr{F}, \omega_{X}^{\cdot}\right)\right)_{x} \neq 0, \quad \text { and } \quad\left(\mathcal{E} \chi t_{X}^{-t}\left(\mathscr{F}, \omega_{X}\right)\right)_{x} \neq 0,
$$

Proof. We may obviously assume that $\mathscr{F} \neq 0$. Localization is exact and commutes with the $\mathcal{H}$ om functor, so $\left(\mathcal{E} \chi t_{X}^{-i}\left(\mathscr{F}, \omega_{X}^{\bullet}\right)\right)_{x} \simeq \mathcal{E} \chi t_{X_{x}}^{-i}\left(\mathscr{F}_{x},\left(\omega_{X}^{\bullet}\right)_{x}\right)$ and then the latter group is isomorphic to $\mathcal{E} t_{X_{x}}^{\operatorname{dim} x-i}\left(\mathscr{F}_{x}, \omega_{X_{x}}^{\cdot}\right)$. This is the Matlis dual of $H_{x}^{i-\operatorname{dim} x}(Y, \mathscr{F})$ by [Har66, V.6.2]. Therefore we obtain that

$$
\left(\mathcal{E x t}_{X}^{-i}\left(\mathscr{F}, \omega_{X}^{\cdot}\right)\right)_{x}=0 \quad \Leftrightarrow \quad H_{x}^{i-\operatorname{dim} x}(Y, \mathscr{F})=0
$$

and since both $\operatorname{depth}_{x} \mathscr{F}$ and $\operatorname{dim}_{x} \mathscr{F}$ remain the same over $Y$, the statement follows from Grothendieck's theorem [BH93, 3.5.7].

Corollary 3.3. Under the same conditions and using the same notation as in (3.2) one has that $\mathscr{F}$ is $C M$ at $x \in X$ if and only if

$$
\left(\mathcal{E} x t_{X}^{-i}\left(\mathscr{F}, \omega_{X}^{\cdot}\right)\right)_{x}=0 \quad \text { for } i \neq d=\operatorname{dim}_{x} \mathscr{F}+\operatorname{dim} x .
$$

Corollary 3.4. Let $(X, D)$ be a rational pair. Then $\mathscr{O}_{X}(-D)$ is a $C M$ sheaf.

Proof. Consider a rational $\log$ resolution $\phi:(\widetilde{X}, \widetilde{D}) \rightarrow(X, D)$ (cf. (2.5)). Using the assumption and Grothendieck duality we obtain the following:

$$
\begin{aligned}
\mathcal{R H o m}_{X}\left(\mathscr{O}_{X}(-D), \omega_{X}^{\cdot}\right) \simeq_{\mathrm{qis}} \mathcal{R} \mathcal{H} \operatorname{Hom}_{X}\left(\mathcal{R} \phi_{*} \mathscr{O}_{\widetilde{X}}(-\widetilde{D}), \omega_{X}\right) \simeq_{\mathrm{qis}} \\
\simeq_{\mathrm{qis}} \mathcal{R} \phi_{*} \mathcal{R} \mathcal{H o m}_{\widetilde{X}}\left(\mathscr{O}_{\widetilde{X}}(-\widetilde{D}), \omega_{\tilde{X}}\right) \simeq_{\mathrm{qis}} \mathcal{R} \phi_{*} \omega_{\tilde{X}}(\widetilde{D}) \simeq_{\mathrm{qis}} \phi_{*} \omega_{\widetilde{X}}(\widetilde{D})[\operatorname{dim} X] .
\end{aligned}
$$

This implies that

$$
\operatorname{Ext}_{X}^{-i}\left(\mathscr{O}_{X}(-D), \omega_{X}\right)=0 \quad \text { for } i \neq \operatorname{dim} X
$$

and hence the statement follows by (3.3).

Corollary 3.5. Let $(X, \Delta)$ be a dlt pair. Then for any effective integral divisor $D \leq\lfloor\Delta\rfloor \mathscr{O}_{X}(-D)$ is a $C M$ sheaf. 
Proof. By [KK09, Thm. 111] $(X, D)$ is a rational pair and hence the statement follows from (3.4).

\section{IRRATIONAL CENTERS}

Definition 4.1. Let $\mathscr{F}$ be a coherent sheaf on a scheme $X$. Then $x \in X$ is an associated point of $\mathscr{F}$ if the maximal ideal $\mathfrak{m}_{X, x} \subset \mathscr{O}_{X, x}$ is an associated prime of the module $\mathscr{F}_{x}$. In other words, $x \in X$ is an associated point of $\mathscr{F}$ if the maximal ideal $\mathfrak{m}_{X, x} \subset \mathscr{O}_{X, x}$ consists of zero-divisors of the module $\mathscr{F}_{x}$.

Now we are ready to make the definition of the namesake of the present article:

Definition 4.2. Let $(X, D)$ be a reduced pair and $\phi:(\widetilde{X}, \widetilde{D}) \rightarrow(X, D)$ a $\log$ resolution. If $x \in X$ is an associated point of $\mathcal{R}^{i} \phi_{*} \mathscr{O}_{\widetilde{X}}(-\widetilde{D})$ for some $i>0$, then we call $Z=\overline{\{x\}}$, the Zariski closure of $\{x\}$, a relative irrational center of $(X, D)$ with respect to $\phi$. A closed subset $Z \subseteq X$ is called an irrational center of $(X, D)$ if there exists a $\log$ resolution $\phi:(\widetilde{X}, \widetilde{D}) \rightarrow(X, D)$ such that $Z$ is a relative irrational center of $(X, D)$ with respect to $\phi$ and $Z \subseteq X$ is called an absolute irrational center if for any $\log$ resolution $\phi:(\widetilde{X}, \widetilde{D}) \rightarrow(X, D), Z$ is a relative irrational center of $(X, D)$ with respect to $\phi$.

REMARK 4.3. If $D=0$ then these definitions coincide and agree with the one given in [AH09]. This can be proven the same way as one proves that having rational singularities does not depend on the resolution chosen.

\section{DEPTH Estimates}

\section{A. Truncated functors and distinguished triangles}

Let $\phi:(\widetilde{X}, \widetilde{D}) \rightarrow(X, D)$ be a $\log$ resolution.

Now define a series of derived category objects recursively as follows. Let $\mathcal{R}^{\geq 0} \phi_{*}:=\mathcal{R} \phi_{*}$ and consider the natural transformation

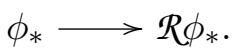


Then let $\mathcal{R}^{\geq 1} \phi_{*} \mathscr{O}_{\widetilde{X}}(-\widetilde{D})$ be defined as the object completing the induced natural morphism to a distinguished triangle:

$$
\phi_{*} \mathscr{O}_{\widetilde{X}}(-\widetilde{D}) \longrightarrow \mathcal{R}_{*} \mathscr{O}_{\widetilde{X}}(-\widetilde{D}) \longrightarrow \mathcal{R}^{\geq 1} \phi_{*} \mathscr{O}_{\widetilde{X}}(-\widetilde{D})[-1] \stackrel{+1}{\longrightarrow} .
$$

By construction there exists a natural morphism,

$$
\mathcal{R}^{1} \phi_{*} \mathscr{O}_{\widetilde{X}}(-\widetilde{D}) \longrightarrow R^{\geq 1} \phi_{*} \mathscr{O}_{\widetilde{X}}(-\widetilde{D}),
$$

and we let $\mathcal{R}^{\geq 2} \phi_{*} \mathscr{O}_{\widetilde{X}}(-\widetilde{D})$ be defined as the object completing the above natural morphism to a distinguished triangle and so on to obtain a series of objects and distinguished triangles for each $p \in \mathbb{N}$ :

$$
\mathcal{R}^{p} \phi_{*} \mathscr{O}_{\widetilde{X}}(-\widetilde{D}) \longrightarrow \mathcal{R}^{\geq p} \phi_{*} \mathscr{O}_{\widetilde{X}}(-\widetilde{D}) \longrightarrow \mathcal{R}^{\geq p+1} \phi_{*} \mathscr{O}_{\widetilde{X}}(-\widetilde{D})[-1] \stackrel{+1}{\longrightarrow} .
$$

\section{B. Easy vanishing theorems for $\mathcal{E} x t$ sheaves}

Lemma 5.1. Let $(X, D)$ be a pair and $\phi:(\widetilde{X}, \widetilde{D}) \rightarrow(X, D)$ a log resolution. Let $p>0$ be a positive integer and $x \in X$ which is not an associated point of $\mathcal{R}^{p} \phi_{*} \mathscr{O}_{\widetilde{X}}(-\widetilde{D})$. Then

$$
\left(\mathcal{E} x t_{X}^{j}\left(\mathcal{R}^{p} \phi_{*} \mathscr{O}_{\widetilde{X}}(-\widetilde{D}), \omega_{X}\right)\right)_{x}=0, \quad \text { for and } j \geq-\operatorname{dim} x \text {. }
$$

Proof. As $x \in X$ is not an associated point of $\mathcal{R}^{p} \phi_{*} \mathscr{O}_{\widetilde{X}}(-\widetilde{D})$, it follows that either $\left(\mathcal{R}^{p} \phi_{*} \mathscr{O}_{\widetilde{X}}(-\widetilde{D})\right)_{x}=0$ or $\operatorname{depth}_{x} \mathcal{R}^{p} \phi_{*} \mathscr{O}_{\widetilde{X}}(-\widetilde{D}) \geq 1$. In the latter case the statement follows from (3.2).

Corollary 5.2. Let $(X, D)$ be a pair and $\phi:(\widetilde{X}, \widetilde{D}) \rightarrow(X, D)$ a log resolution. Let $p>0$ be a positive integer and $x \in X$ which is not an associated point of $\mathcal{R}^{i} \phi_{*} \mathscr{O}_{\widetilde{X}}(-\widetilde{D})$ for any $i \geq p$. Then

$$
\left(\mathcal{E} x t_{X}^{j}\left(\mathcal{R}^{\geq p} \phi_{*} \mathscr{O}_{\widetilde{X}}(-\widetilde{D}), \omega_{X}^{\cdot}\right)\right)_{x}=0, \quad \text { for } j \geq-\operatorname{dim} x .
$$

Proof. If $p \gg 0$ then $\mathcal{R}^{p} \phi_{*}=\mathcal{R}^{\geq p} \phi_{*}=0$, and hence the statement holds trivially. Next apply the functor $\mathcal{R} \mathcal{H} m_{X}\left(\ldots, \omega_{X}^{*}\right)$ to the distinguished triangle:

$$
\mathcal{R}^{p} \phi_{*} \mathscr{O}_{\widetilde{X}}(-\widetilde{D}) \longrightarrow \mathcal{R}^{\geq p} \phi_{*} \mathscr{O}_{\widetilde{X}}(-\widetilde{D}) \longrightarrow \mathcal{R}^{\geq p+1} \phi_{*} \mathscr{O}_{\widetilde{X}}(-\widetilde{D})[-1] \stackrel{+1}{\longrightarrow}
$$

This is essentially the truncated complex of $\mathcal{R} \phi_{*} \mathscr{O}_{\widetilde{X}}(-\widetilde{D})$. The shift is included to make the subsequent definitions simpler and more balanced. 
to obtain the long exact sequence,

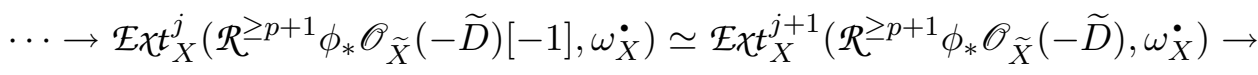

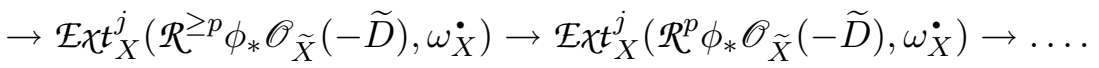

Now the statement follows from (5.1) by descending induction on $p$.

\section{C. Main theorem}

Theorem 5.3. Let $(X, D)$ be a reduced pair, $\phi:(\widetilde{X}, \widetilde{D}) \rightarrow(X, D)$ a log resolution, and $x \in X$ which is not the general point of a relative irrational center of $(X, D)$ with respect to $\phi$. Then

$$
\left(\mathcal{E} \chi t_{X}^{-i}\left(\phi_{*} \mathscr{O}_{\widetilde{X}}(-\widetilde{D}), \omega_{X}^{\cdot}\right)\right)_{x}=0, \quad \text { for } i<\min \left(3, \operatorname{codim}_{X} x\right)+\operatorname{dim} x,
$$

in particular

$$
\operatorname{depth}_{x} \phi_{*} \mathscr{O}_{\widetilde{X}}(-\widetilde{D}) \geq \min \left(3, \operatorname{codim}_{X} x\right)
$$

Proof. First observe that $\operatorname{dim}_{x} \phi_{*} \mathscr{O}_{\widetilde{X}}(-\widetilde{D})=\operatorname{codim}_{X} x$, so (5.3.1) implies (5.3.2) by (3.2). I will prove (5.3.1). Consider the distinguished triangle:

$$
\phi_{*} \mathscr{O}_{\widetilde{X}}(-\widetilde{D}) \longrightarrow \mathcal{R} \phi_{*} \mathscr{O}_{\widetilde{X}}(-\widetilde{D}) \longrightarrow \mathcal{R}^{\geq 1} \phi_{*} \mathscr{O}_{\widetilde{X}}(-\widetilde{D})[-1] \stackrel{+1}{\longrightarrow},
$$

and the long exact sequence it induces:

$$
\begin{aligned}
\cdots \rightarrow \mathcal{E} \chi t_{X}^{-i}\left(\mathcal{R} \phi_{*} \mathscr{O}_{\widetilde{X}}(-\widetilde{D}), \omega_{X}^{\cdot}\right) \rightarrow & \mathcal{E x t}_{X}^{-i}\left(\phi_{*} \mathscr{O}_{\widetilde{X}}(-\widetilde{D}), \omega_{X}^{\cdot}\right) \rightarrow \\
& \rightarrow \mathcal{E x t}_{X}^{-i+2}\left(\mathcal{R}^{\geq 1} \phi_{*} \mathscr{O}_{\widetilde{X}}(-\widetilde{D}), \omega_{X}\right) \rightarrow \ldots
\end{aligned}
$$

If $i<3+\operatorname{dim} x$, then $-i+2 \geq-\operatorname{dim} x$ so $\left(\mathcal{E} x t_{X}^{-i+2}\left(\mathcal{R}^{\geq 1} \phi_{*} \mathscr{O}_{\widetilde{X}}(-\widetilde{D}), \omega_{X}\right)\right)_{x}=0$

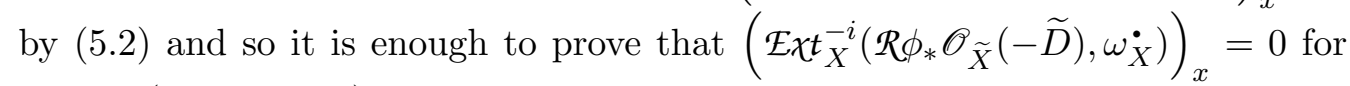
$i<\min \left(3, \operatorname{codim}_{X} x\right)$.

Let $n=\operatorname{dim} X$ and observe that

$$
\begin{aligned}
& \operatorname{Ext}_{X}^{-i}\left(\mathcal{R} \phi_{*} \mathscr{O}_{\widetilde{X}}(-\widetilde{D}), \omega_{X}\right) \simeq h^{-i}\left(\mathcal{R} \mathcal{H} m_{X}\left(\mathcal{R} \phi_{*} \mathscr{O}_{\widetilde{X}}(-\widetilde{D}), \omega_{X}\right)\right) \simeq \\
& \simeq h^{-i}\left(\mathcal{R} \phi_{*} \mathcal{R H o m}_{\widetilde{X}}\left(\mathscr{O}_{\widetilde{X}}(-\widetilde{D}), \omega_{\tilde{X}}\right)\right) \simeq h^{-i}\left(\mathcal{R} \phi_{*} \omega_{\widetilde{X}}(\widetilde{D})\right) \simeq \mathcal{R}^{n-i} \phi_{*} \omega_{\widetilde{X}}(\widetilde{D})
\end{aligned}
$$

by Grothendieck duality and the fact that $\widetilde{X}$ is smooth and $\widetilde{D}$ is a Cartier divisor. Now observe that if $i<n$, then $\left(\mathcal{R}^{n-i} \phi_{*} \omega_{\widetilde{X}}(\widetilde{D})\right)_{x}=0$ by $(2.9)$. 
Corollary 5.4. Let $(X, D)$ be a normal pair and $x \in X$ which is not the general point of an absolute irrational center of $(X, D)$. Then

$$
\operatorname{depth}_{x} \mathscr{O}_{X}(-D) \geq \min \left(3, \operatorname{codim}_{X} x\right)
$$

Proof. Let $\phi:(\widetilde{X}, \widetilde{D}) \rightarrow(X, D)$ be a $\log$ resolution for which $x \in X$ is not the general point of a relative irrational center of $(X, D)$ with respect to $\phi$. Notice that as $(X, D)$ is normal, by definition,

$$
\mathscr{O}_{X}(-D) \simeq \phi_{*} \mathscr{O}_{\widetilde{X}}(-\widetilde{D})
$$

so the statement is straightforward from (5.3).

\section{Applications to log canonical pairs}

The key point of applying the results of this paper to log canonical pairs is that absolute irrational centers are non-klt centers. It is easy to see that the union of all non-klt centers of a log canonical pair contains the locus where that log canonical pair is not rational and hence it contains the union of all absolute irrational centers. However, I am claiming that there is a closer relationship, namely that the absolute irrational centers themselves are non-klt centers.

Next we will discuss the key step in applying the theory of irrational centers to $\log$ canonical pairs. We will use the following slight abuse of notation: For a $\log$ resolution $\phi:\left(Y, \Delta_{Y}\right) \rightarrow(X, \Delta)$ we will denote the log resolution $\phi:\left(Y,\left\lfloor\Delta_{Y}\right\rfloor\right) \rightarrow(X,\lfloor\Delta\rfloor)$ by the same symbol. This makes sense as $\phi$ really stands for the birational morphism $\phi: Y \rightarrow X$ that, as a morphism, is a priori independent of the choice of boundary divisor.

We will need the following in the proof.

Definition 6.1. Let $(Z, \Theta)$ be a dlt pair. A $\log$ resolution of $(Z, \Theta)$, $g:(Y, \Gamma) \rightarrow(Z, \Theta)$ is called a Szabó-resolution, if there exist $A, B$ effective $\mathbb{Q}$-divisors on $Y$ without common irreducible components, such that $\operatorname{supp}(A+B) \subset \operatorname{Exc}(g),\lfloor A\rfloor=0$, and

$$
K_{Y}+\Gamma \sim_{\mathbb{Q}} g^{*}\left(K_{Z}+\Theta\right)-A+B .
$$

Remark 6.2. Every dlt pair admits a Szabó-resolution by [Sza94] (cf. [KM98, $2.44])$. 
Theorem 6.3. Let $(X, \Delta)$ be a log canonical pair. Then there exists a log resolution $\phi:\left(Y, \Delta_{Y}\right) \rightarrow(X, \Delta)$ such that every relative irrational center of $(X,\lfloor\Delta\rfloor)$ with respect to $\phi$ is the image of a non-klt center of $\left(Y, \Delta_{Y}\right)$. In particular, every absolute irrational center of $(X,\lfloor\Delta\rfloor)$ is also a non-klt center of $(X, \Delta)$.

Proof. First let $\psi:\left(Z, \Delta_{Z}+E\right) \rightarrow(X, \Delta)$ be a minimal dlt model [KK10, 3.1], where $\Delta_{Z}=\psi_{*}^{-1} \Delta$ and $E=\operatorname{Exc}(\psi)$. By the definition of the minimal dlt model $Z$ is $\mathbb{Q}$-factorial and

$$
K_{Z}+\Delta_{Z}+E \sim_{\mathbb{Q}} \psi^{*}\left(K_{X}+\Delta\right) .
$$

Next let $\eta:\left(Y, \Delta_{Y}+E_{Y}\right) \rightarrow\left(Z, \Delta_{Z}+E\right)$ be a Szabó-resolution (6.1), where $\Delta_{Y}=\eta_{*}^{-1} \Delta_{Z}$ and $E_{Y}=\eta_{*}^{-1} E$. Then there are $A, B$ effective $\mathbb{Q}$-divisors on $Y$ without common irreducible components, such that $\operatorname{supp}(A+B) \subset \operatorname{Exc}(\eta)$, $\lfloor A\rfloor=0$, and

$$
K_{Y}+\Delta_{Y}+E_{Y} \sim_{\mathbb{Q}} \eta^{*}\left(K_{Z}+\Delta_{Z}+E\right)-A+B .
$$

Let $B_{\varepsilon}:=B+\varepsilon\left(\eta^{*} E-E_{Y}\right)$ where $0<\varepsilon \ll 1$. It follows that

$$
\begin{aligned}
\left\lceil B_{\varepsilon}\right\rceil-\left\lfloor\Delta_{Y}\right\rfloor \sim_{\mathbb{Q}} K_{Y}+\left\{\Delta_{Y}\right\}+(1-\varepsilon) E_{Y}+A+\left\{-B_{\varepsilon}\right\}+ & \\
& -\eta^{*}\left(K_{Z}+\Delta_{Z}+(1-\varepsilon) E\right),
\end{aligned}
$$

and then the relative Kawamata-Viehweg vanishing theorem implies that

$$
\mathcal{R}^{i} \eta_{*} \mathscr{O}\left(\left\lceil B_{\varepsilon}\right\rceil-\left\lfloor\Delta_{Y}\right\rfloor\right)=0 \quad \text { for } i>0 .
$$

As $B_{\varepsilon}$ is still an effective $\eta$-exceptional divisor, it follows that

$$
\eta_{*} \mathscr{O}_{Y}\left(\left\lceil B_{\varepsilon}\right\rceil-\left\lfloor\Delta_{Y}\right\rfloor\right) \simeq \mathscr{O}_{Z}\left(-\left\lfloor\Delta_{Z}\right\rfloor\right),
$$

cf. (2.4), and hence

$$
\mathcal{R} \eta_{*} \mathscr{O}_{Y}\left(\left\lceil B_{\varepsilon}\right\rceil-\left\lfloor\Delta_{Y}\right\rfloor\right) \simeq_{\text {qis }} \mathscr{O}_{Z}\left(-\left\lfloor\Delta_{Z}\right\rfloor\right) .
$$

Clearly, $\phi=\psi \circ \eta$ is a $\log$ resolution of $(X, \Delta)$ and $\Delta_{Y}=\phi_{*}^{-1} \Delta$. Then from (6.3.1) and (6.3.3) we obtain

$\left\lceil B_{\varepsilon}\right\rceil-\left\lfloor\Delta_{Y}\right\rfloor \sim_{\mathbb{Q}} K_{Y}+\left\{\Delta_{Y}\right\}+E_{Y}+A+\left\{-B_{\varepsilon}\right\}+\varepsilon\left(\eta^{*} E-E_{Y}\right)-\phi^{*}\left(K_{X}+\Delta\right)$.

Note that as opposed to (6.3.3), here the boundary relative to $\phi$ may have components with coefficient 1 , namely $E_{Y}$, so we cannot apply the relative KawamataViehweg vanishing theorem with respect to $\phi$. 
On the other hand, notice that since $\left(Z, \Delta_{Z}+E\right)$ is $\mathbb{Q}$-factorial and dlt, so is $\left(Z, \Delta_{Z}\right)$ and then by $[\operatorname{Kov} 10,2.1] \mathcal{R}^{i} \eta_{*} \mathscr{O}_{Y}\left(-\left\lfloor\Delta_{Y}\right\rfloor\right)=0$ for $i>0$. Therefore, using (6.3.4), we obtain that

$$
\begin{aligned}
\mathcal{R} \phi_{*} \mathscr{O}_{Y}\left(-\left\lfloor\Delta_{Y}\right\rfloor\right) & \simeq_{\mathrm{qis}} \mathcal{R} \psi_{*} \mathcal{R} \eta_{*} \mathscr{O}_{Y}\left(-\left\lfloor\Delta_{Y}\right\rfloor\right) \simeq_{\mathrm{qis}} \mathcal{R} \psi_{*} \mathscr{O}_{Z}\left(-\left\lfloor\Delta_{Z}\right\rfloor\right) \simeq_{\mathrm{qis}} \\
& \simeq_{\mathrm{qis}} \mathcal{R} \psi_{*} \mathcal{R} \eta_{*} \mathscr{O}_{Y}\left(\left\lceil B_{\varepsilon}\right\rceil-\left\lfloor\Delta_{Y}\right\rfloor\right) \simeq_{\mathrm{qis}} \mathcal{R} \phi_{*} \mathscr{O}_{Y}\left(\left\lceil B_{\varepsilon}\right\rceil-\left\lfloor\Delta_{Y}\right\rfloor\right) .
\end{aligned}
$$

Next use Kollár-Ambro torsion freeness: Applying [Fuj09, 2.39] to (6.3.5) we obtain that the closure of any associated point of

$$
\mathcal{R}^{i} \phi_{*} \mathscr{O}_{Y}\left(-\left\lfloor\Delta_{Y}\right\rfloor\right) \simeq \mathcal{R}^{i} \phi_{*} \mathscr{O}_{Y}\left(\left\lceil B_{\varepsilon}\right\rceil-\left\lfloor\Delta_{Y}\right\rfloor\right)
$$

for some $i>0$ is a non-klt center of $(X, \Delta)$. Similarly, the closure of an associated point of $\mathcal{R}^{i} \phi_{*} \omega_{Y}\left(\left\lfloor\Delta_{Y}\right\rfloor\right)$ for some $i>0$ is a non-klt center of $(X, \Delta)$ by [Fuj09, 2.39].

As indicated in the introduction we obtain the following corollary:

Corollary 6.4. Let $(X, \Delta)$ be a log canonical pair and $x \in X$ which is not the general point of a non-klt center of $(X, \Delta)$. Then

$$
\operatorname{depth}_{x} \mathscr{O}_{X}(-\lfloor\Delta\rfloor) \geq \min \left(3, \operatorname{codim}_{X} x\right) .
$$

Proof. By (6.3) there exists a $\log$ resolution $\phi:\left(Y, \Delta_{Y}\right) \rightarrow(X, \Delta)$ such that $x$ is not a relative irrational center of $(X,\lfloor\Delta\rfloor)$ with respect to $\phi$. Then

$$
\operatorname{depth}_{x} \phi_{*} \mathscr{O}_{Y}\left(-\left\lfloor\Delta_{Y}\right\rfloor\right) \geq \min \left(3, \operatorname{codim}_{X} x\right)
$$

by $(5.3)$ and $\mathscr{O}_{X}(-\lfloor\Delta\rfloor) \simeq \phi_{*} \mathscr{O}_{Y}\left(-\left\lfloor\Delta_{Y}\right\rfloor\right)$ by $(2.4)$.

Acknowledgment. I would like to thank Valery Alexeev, Zsolt Patakfalvi, and Karl Schwede for helpful comments.

\section{REFERENCES}

[Ale08] V. Alexeev: Limits of stable pairs, Pure Appl. Math. Q. 4 (2008), no. 3, Special Issue: In honor of Fedor Bogomolov. Part 2, 767-783. 2435844 (2009j:14020)

[AH09] V. AleXeEv And C. D. HACON: Non-rational centers of log canonical singularities, in preparation, 2009.

[Amb03] F. Ambro: Quasi-log varieties, Tr. Mat. Inst. Steklova 240 (2003), no. Biratsion. Geom. Linein. Sist. Konechno Porozhdennye Algebry, 220-239. MR1993751 (2004f:14027) 
[BH93] W. Bruns And J. Herzog: Cohen-Macaulay rings, Cambridge Studies in Advanced Mathematics, vol. 39, Cambridge University Press, Cambridge, 1993. MR1251956 (95h:13020)

[Con00] B. ConRaD: Grothendieck duality and base change, Lecture Notes in Mathematics, vol. 1750, Springer-Verlag, Berlin, 2000. MR1804902 (2002d:14025)

[DB81] P. Du BoIs: Complexe de de Rham filtré d'une variété singulière, Bull. Soc. Math. France 109 (1981), no. 1, 41-81. MR613848 (82j:14006)

[Fuj09] O. FujINo: Introduction to the log minimal model program for log canonical pairs, version 6.01, 2009. arXiv:0907.1506v1 [math.AG]

[GNPP88] F. Gulllén, V. Navarro Aznar, P. Pascual Gainza, and F. Puerta: Hyperrésolutions cubiques et descente cohomologique, Lecture Notes in Mathematics, vol. 1335, Springer-Verlag, Berlin, 1988, Papers from the Seminar on Hodge-Deligne Theory held in Barcelona, 1982. MR972983 (90a:14024)

[HK10] C. D. HACON AND S. J. KOVÁCS: Classification of higher dimensional algebraic varieties, Oberwolfach Seminars, Birkhäuser Boston, Boston, MA, 2010.

[Har66] R. HARTSHORNE: Residues and duality, Lecture notes of a seminar on the work of A. Grothendieck, given at Harvard 1963/64. With an appendix by P. Deligne. Lecture Notes in Mathematics, No. 20, Springer-Verlag, Berlin, 1966. MR0222093 (36 \#5145)

[Kaw02] T. KAWASAKI: On arithmetic Macaulayfication of Noetherian rings, Trans. Amer. Math. Soc. 354 (2002), no. 1, 123-149 (electronic). MR1859029 (2002i:13001)

[Kol10] J. Kollár: A local version of the Kawamata-Viehweg vanishing theorem, preprint, 2010. arXiv:1005.4843v1 [math.AG]

[KK09] J. Kollár and S. J. KovÁcs: Rational pairs, preprint, 2009.

[KK10] J. Kollár And S. J. KovÁcs: Log canonical singularities are Du Bois, J. Amer. Math. Soc. 23 (2010), no. 3, 791-813. doi:10.1090/S0894-0347-10-00663-6

[KM98] J. Kollár AND S. Mori: Birational geometry of algebraic varieties, Cambridge Tracts in Mathematics, vol. 134, Cambridge University Press, Cambridge, 1998, With the collaboration of C. H. Clemens and A. Corti, Translated from the 1998 Japanese original. MR1658959 (2000b:14018)

[Kov00] S. J. KovÁcs: A characterization of rational singularities, Duke Math. J. 102 (2000), no. 2, 187-191. MR1749436 (2002b:14005)

[Kov10] S. J. KovÁcs: DB pairs and vanishing theorems, Journal of Mathematics of Kyoto University, Nagata Memorial Issue (2010), 21 pages.

[ST08] K. Schwede And S. TAKAGI: Rational singularities associated to pairs, Michigan Math. J. 57 (2008), 625-658, Special volume in honor of Melvin Hochster. MR2492473

[Sza94] E. Szabó: Divisorial log terminal singularities, J. Math. Sci. Univ. Tokyo 1 (1994), no. 3, 631-639. MR1322695 (96f:14019)

Sándor J Kovács

University of Washington, Department of Mathematics, Box 354350, Seattle, WA 98195-4350, USA 
E-mail:skovacs@uw.edu

URL: http://www.math.washington.edu/ kovacs 